\title{
FINITE GROUPS WHOSE $n$-MAXIMAL SUBGROUPS ARE SUBNORMAL
}

\author{
BY \\ AVINO'AM MANN
}

Introduction. Dedekind has determined all groups whose subgroups are all normal (see, e.g., [5, Theorem 12.5.4]). Partially generalizing this, Wielandt showed that a finite group is nilpotent, if and only if all its subgroups are subnormal, and also if and only if all maximal subgroups are normal [5, Corollary 10.3.1, 10.3.4]. Huppert [7, Sätze 23, 24] has shown that if all 2nd-maximal subgroups of a finite group are normal, the group is supersolvable, while if all 3rd-maximal subgroups are normal, the group is solvable of rank at most 2 (see below for the definitions of the terms employed). Continuing this, Janko [8] has determined all nonsolvable finite groups, all of whose 4-maximal subgroups are normal, and also proved some results on the structure of solvable groups with the same property. Later, he has also determined all finite simple groups, all of whose 5-maximal subgroups are normal [9].

The present paper deals with similar problems. Our main concern is with the class of groups defined in the title. In $\$ 2$, we derive some simple properties of these groups. For $n \leqq 5$, we extend the aforementioned results of Huppert and Janko to cover also our case (i.e., replacing normality by subnormality), and prove some results under slightly more general assumptions. In $\$ 3$ we study solvable groups with subnormal $n$-maximal subgroups. If the order of the group in question has enough distinct prime divisors, the structure of the group is very limited. Thus, if the number of these primes exceeds $n$, the group is nilpotent, while if it is equal to $n$, we can still completely determine our groups. If the number is $n-1$, the group has a Sylow tower. Then, imposing the stronger requirement that $n$-maximal subgroups are quasi-normal, we find that the rank of the group cannot exceed $n-1$. In the last section we allow again our groups to be nonsolvable. The main result here states, that if each $n$-maximal subgroup is subnormal, and if the order of the groups has a large number of distinct prime divisors then the group is, in fact, solvable. Here, "large" is not only with respect to $n$, but also with respect to the number of prime divisors of the indices of maximal subgroups of subgroups.

Most of the contents of this paper formed a part of the author's Ph.D. thesis prepared in the Hebrew University, Jerusalem, under the supervision of Professor S. A. Amitsur. I would like to take this opportunity to thank Professor Amitsur for his interest and encouragement, as well as for a number of valuable suggestions.

Received by the editors March 9, 1967. 
1. Definitions and notations. If $G$ is a group, a chain $H_{0} \subset H_{1} \subset \ldots \subset H_{n}=G$ is a maximal chain if each $H_{i}$ is a maximal subgroup of $H_{i+1}$. The subgroup $H_{0}$ in such a series is an $n$-maximal subgroup. A subgroup $H$ of $G$ is at least $n$-maximal, if it is $m$-maximal for some $m \geqq n$. A subgroup $H$ is quasi-normal, if it permutes with all other subgroups; quasi-normal subgroups of finite groups are subnormal [14, Theorem 19, p. 438]. If $G$ is a solvable finite group, the rank of $G$, denoted $r(G)$, is the maximal integer $k$ such that $G$ has a chief factor of order $p^{k}$, for some prime $p$. If $m$ is an integer, $G^{m}$ denotes the subgroup generated by the $m$ th powers of elements of $G$. $G_{p}$, for $p$ a prime, denotes always a Sylow $p$-subgroup of the finite group $G .|G|$ is the order of $G$, and $|G: H|$ is the index of the subgroup $H$ in $G . \Omega(m)$ is the number of factors in a factorization of $m$ as a product of primes, while $w(m)$ is the number of different primes appearing in such a factorization.

Throughout the paper, $G$ denotes a finite group (in $\S 3$ this group is also assumed to be solvable), and $n$ denotes a fixed natural number.

\section{Some lemmata and solvability criteria.}

LEMMA 1. If each n-maximal subgroup of $G$ is subnormal, then each $(n-1)$ maximal subgroup is nilpotent.

Proof. Let $H$ be an (n-1)-maximal subgroup, and let $K$ be a maximal subgroup of $H$. Then $K$ is $n$-maximal in $G$, hence subnormal, and so it is certainly subnormal in $H$. However, for maximal subgroups subnormality is equivalent to normality. Therefore each maximal subgroup of $H$ is normal in it, and $H$ is nilpotent.

LEMMA 2. Let each n-maximal subgroup of $G$ be subnormal, and let $k \geqq n$. Then each $k$-maximal subgroup of $G$ is subnormal.

Proof. Let $H$ be a $k$-maximal subgroup of $G$, and let $H_{1}$ and $H_{2}$ be an $n$-maximal and an (n-1)-maximal subgroup, respectively, such that $H \subseteq H_{1} \subseteq H_{2}$. By the preceding lemma, $H_{2}$ is nilpotent, and so $H_{1}$ is also nilpotent. Therefore $H$ is subnormal in $H_{1}$, and since $H_{1}$ is subnormal in $G$, by assumption, $H$ is also subnormal in $G$.

Lemma 3. Let $F(G)$ be the Fitting subgroup of $G$. Each n-maximal subgroup is subnormal, if and only if each n-maximal subgroup is contained in $F(G)$. In particular, in these circumstances each n-maximal subgroup of $G / F(G)$ is trivial.

Proof. Suppose each $n$-maximal subgroup of $G$ is subnormal, and let $H$ be such a subgroup. Then Lemma 1 implies that $H$ is a nilpotent subnormal subgroup, and therefore contained in $F(G)$. Conversely, if any subgroup $K$ of $G$ is contained in $F(G)$, it is subnormal in the nilpotent subgroup $F(G)$, and since $F(G) \triangleleft G$, also in $G$.

THEOREM 1. If each maximal subgroup $M$ of $G$ contains a subgroup $M_{1}$ that is subnormal in $G$ and is either maximal in $M$ or equal to it, then $G^{\prime}$ is nilpotent. 
If $G$ is any finite group, and $H$ is any subgroup of $G$, we denote by $H_{G}$ and $H_{S G}$ the maximal normal and subnormal, respectively, subgroups of $G$ contained in $H$.

Lemma 4. If $M$ is maximal in $G$, then $M_{G}=M_{S G}$.

Proof. Obviously $M_{G} \subseteq M_{S G}$. Let $a \in M$; then $M_{S G}^{a} \subseteq M$ and $M_{S G}^{a} \triangleleft \triangleleft G$, therefore $M_{S G}^{a} \subseteq M_{S G}$ and $M_{S G}$ is normal in $M$. Let $M_{S G} \triangleleft M_{1} \triangleleft \cdots \triangleleft M_{k}=G$ be any normal chain between $M_{S G}$ and $G$. Then $M_{1} \triangleleft \triangleleft G$, and by maximality of $M_{S G}, M_{1} \nsubseteq M$. By maximality of $M, G=\left\{M, M_{1}\right\}$. Also, $M_{S G} \triangleleft M$ and $M_{S G} \triangleleft M_{1}$; therefore $M_{S G} \triangleleft G$ and $M_{S G} \subseteq M_{G}$.

Proof of Theorem 1. Let $M$ be any maximal subgroup of $G$, and $M_{1}$ its subgroup given by the conditions of the theorem. Then $M_{S G}=M_{1}$ or $M_{S G}=M$, because $M_{1} \subseteq M_{S G}$ and $M_{1}$ is maximal. In any case, $M / M_{S G}$ is cyclic (of prime order). Now the nilpotence of $G^{\prime}$ follows from Lemma 4 and a theorem of Baer [1, Corollary 2, p. 159], according to which $G^{\prime}$ is nilpotent for any finite group $G$, if and only if $M / M_{G}$ is abelian for each maximal subgroup $M$ of $G$.

Next, we consider finite groups satisfying the following condition:

(P). If $M$ is a maximal subgroup of $G$, there exists a subgroup $M_{1}$, subnormal in $G$, such that $M_{1}=M, M_{1}$ is maximal in $M$, or $M_{1}$ is 2nd-maximal in $M$.

THEOREM 2. A finite simple (nonabelian) group satisfying $(\mathrm{P})$ is isomorphic to $A_{5}$.

Proof. Let $M$ be any maximal subgroup of $G$, and $M_{1}$ the subgroup given by condition (P). Since $G$ is simple, and $M_{1}$ is subnormal, $M_{1}=\{1\}$.

If $M_{1}=M$, we obtain $M=1$.

If $M_{1}$ is maximal in $M, M$ must be cyclic of prime order.

Suppose now $M_{1}$ is 2 nd-maximal in $M$. Then there exists a chain $\{1\} \subset L \subset M$, with $\{1\}$ maximal in $L$ and $L$ maximal in $M . L$ is then cyclic of prime order $p$, say. Since $M$ has an abelian maximal subgroup, it is solvable [6].

If $L \triangleleft M, L$ has a prime index in $M$ and $|M|=p q, q$ a prime ( $p=q$ is possible). Any proper subgroup of such an $M$ is cyclic.

If $L \bowtie M$, let $K$ be a minimal normal subgroup of $M$, then $K$ is elementary abelian of order $q^{i}$, for a suitable prime $q$. By maximality of $L, M=K L$ and $|m|=p q^{i}$. $p \neq q$, because otherwise $M$ would be a $p$-group and $L$ would be normal in $M$.

Any proper subgroup of $M$ is either of order $p$, and cyclic, or of order $q^{j}$, and contained in $K$, and so abelian. Subgroups of order $p q^{j}$ do not exist, because such a subgroup would contain a $p$-Sylow subgroup of $M$, which is conjugate to $L$, and thus contradict the maximality of $L$.

In all cases it turns out that each proper subgroup of $M$ is abelian, and so each 2nd-maximal subgroup of $G$ is abelian. By a theorem of Suzuki [16], $G \cong A_{5}$.

The group SL $(2,5)$ also satisfies $(\mathrm{P})$. We do not know whether there are nonsolvable (P) groups apart from $A_{5}$ and $\operatorname{SL}(2,5)$.

We note also the following fact about $(\mathrm{P})$ groups. 
LEMMA 5. Under the assumption (and notations) of (P), $M_{S G}$ is either equal or maximal in $M$, or $M_{S G}=M_{1}$.

Proof. Suppose $M_{S G} \neq M$ and $M_{S G}$ is not maximal in $M$. Let $M_{1} \subset L \subset M$, with $M_{1}$ maximal in $L$ and $L$ maximal in $M$. Now $M_{1}$ is a subnormal maximal subgroup of $L$, and therefore $M_{1} \triangleleft L$ and moreover, $\left|L: M_{1}\right|=p$ is a prime. If $M_{S G} \subseteq L$, then $M_{1} \subseteq M_{S G} \subseteq L$, and because $M_{1}$ is maximal in $L$, while $M_{S G}$ is not maximal in $G$, $M_{1}=M_{S G}$. If $M_{S G} \nsubseteq L$, then $M_{1} \subseteq M_{S G} \cap L \subseteq L$, and $M_{1}=M_{S G} \cap L$ follows as before. Also, $M_{S G} \triangleleft M$ and $L$ is maximal in $M$; therefore $M=M_{S G} L$. Hence $\left|M: M_{S G}\right|$ $=\left|L: L \cap M_{S G}\right|=\left|L: M_{1}\right|=p$, and $M_{S G}$ is maximal in $M$.

THEOREM 3. If each 2nd-maximal subgroup of $G$ is either subnormal, or has a maximal subgroup which is subnormal, then $G^{\prime \prime}$ is nilpotent.

Proof. Obviously, $G$ has property $(P)$. Let $K$ be a maximal normal subgroup of $G$. Then $G / K$ satisfies the same condition as $G$, and also $(P)$. By Theorem $2, G / K$ is either of prime order or isomorphic to $A_{5}$. However, $A_{5}$ has 2nd-maximal subgroups of order 4 , and these violate the conditions of the theorem. Hence, $G / K$ is cyclic, and $G^{\prime} \subseteq K$.

Also, $K$ is a maximal subgroup of $G$, and our assumptions imply that each maximal subgroup of $G$ satisfies the conditions of Theorem 1 . Hence, $K^{\prime}$ is nilpotent, and so $G^{\prime \prime}$ is nilpotent.

COROLlary 1. If each 2nd-maximal subgroup of $G$ is subnormal, or if each $3 r d-$ maximal subgroup is subnormal, then $G$ is solvable.

This result is no longer true for 4-maximal subgroups. As a substitute, we have

THEOREM 4. If each 4-maximal subgroup of $G$ is subnormal, then $G$ is either solvable, or isomorphic to one of the groups $\operatorname{SL}(2,5)$ or $\operatorname{PSL}(2, p)$. Here $p$ is a prime, $p=5$ or $p \equiv \pm 3, \pm 13(40)$, and $p-1$ and $p+1$ are products of at most three prime numbers.

If we substitute "normal" for "subnormal", this result is due to Janko [8, Theorems 1, 2].

Proof. First, suppose $G$ is simple. Then each 4-maximal subgroup is trivial, and our result reduces to Janko's one just mentioned.

Now, let $G$ be nonsolvable and not simple. Let $N$ be a maximal normal subgroup of $G$. Each proper subgroup of $G$ is solvable, by Corollary 1, and therefore $N$ is solvable, and $G / N$ is a nonsolvable simple group. By the preceding paragraph, $G / N \cong \operatorname{PSL}(2, p)$, for some $p$.

For the above values of $p, \operatorname{PSL}(2, p)$ has the four-group as a 2-Sylow subgroup, and this is 2nd-maximal. Let $M / N$ be a 2-Sylow subgroup of $G / N$, and $L / M$ a subgroup of order 2 of $M / N$. $L$ is nilpotent, by Lemma 1 . Let $L_{2}$ be a 2-Sylow subgroup of $L$; then, if $L_{2} \neq L, L_{2}$ is subnormal in $G$, by Lemma 2. But then $L_{2} / N$ is subnormal in $G / N$, which is impossible. So $L$ (and $N$ ) is a 2-group. 
Let $K$ be any 2nd-maximal subgroup of $M . K$ is subnormal in $G$, implying $K N / N$ $\triangleleft \triangleleft G / N$, and therefore $K N / N=1$ and $K \subseteq N$. That means that $N$ is the only 2ndmaximal subgroup of $M$, and it is well known that this implies that $M$ is cyclic or a generalized quaternion group. $M$ cannot be cyclic, because $M / N$ is not cyclic. Therefore $M$ is a generalized quaternion group, and $N$ must be of order 2 (otherwise $M$ has other subgroups of the same order). Being a normal subgroup of order $2, N$ is central in $G$.

Now $N \subseteq Z(G), G / N \cong \operatorname{PSL}(2, q)$ and $G=G^{\prime}$ (otherwise $G^{\prime}$, and also $G$, is solvable) imply, according to a theorem of Schur [15, IX, p. 119], that $G \cong \operatorname{SL}(2, p)$.

Either $p+1$ or $p-1, p+\varepsilon$ say, is of the form $4 s$. If $s=2^{n}$, then $s=1$, or else 8|| $\operatorname{PSL}(2, p) \mid$, which does not hold for our values of $p$. But then $p=3$, or $p=5$, and in the first case $G$ is solvable. If $p \neq 5$ we may assume, then, that $s$ is divisible by an odd prime $q$. PSL $(2, p)$ has a dihedral subgroup of order $p+\varepsilon$, and we can find in it a subgroup of order $q$, and this would be $k$-maximal, $k \geqq 3$. Let $T / N$ be the corresponding subgroup of $G / N$, and let $T_{q}$ be its $q$-Sylow subgroup. Then $T_{q}$ is $(k+1)$-maximal in $G$, so subnormal, and $T / N \triangleleft \triangleleft G / N$, a contradiction. Hence, $p=5$.

Janko [9] has also shown that all simple finite groups having all their 5-maximal subgroups trivial are of the type PSL $(2, q)$. Using this result, one can obtain the following.

THEOREM 5. If each 5-maximal subgroup of $G$ is subnormal, then $G$ is either solvable, or isomorphic to one of the following groups:

I. PSL $(2, q), q=2^{3}, 3^{2}, 3^{3}$ or $q$ is a prime, $q>3, q+1$ and $q-1$ are products of at most four primes.

II. SL $(2, q), q$ a prime, $q>3, q+1$ and $q-1$ are products of three primes at most.

III. PGL $(2, q), q$ as in II, and moreover, $q \neq 7$ and $q \neq \equiv \pm 1$ (10).

IV. PSL $(2, q) \times C_{r}, r$ a prime, $q$ as in III.

V. $\operatorname{SL}(2,5) \times C_{r}, r$ a prime.

VI. One of the two representation groups of $S_{5}$ (the one with generalized quaternion Sylow 2-subgroup; see [15, pp. 121-123]).

We shall not give the proof, which is very similar to the preceding one.

3. Solvable groups with subnormal $n$-maximal subgroups. Throughout this section, $G$ denotes a solvable finite group.

THEOREM 6. If each n-maximal subgroup of $G$ is subnormal, and if $w(|G|) \geqq n+1$, then $G$ is nilpotent.

Proof. Let $P$ be any Sylow subgroup of $G$. Let $P=H_{0} \subset H_{1} \subset \ldots \subset H_{m}=G$ be a chain of subgroups, with each $H_{i}$ maximal in $H_{i+1}$. Since $G$ is solvable, each index $\left|H_{i+1}: H_{i}\right|$ is a prime-power, and since $|G: P|$ is divisible by $n$ distinct primes, $m \geqq n$. However, $P$ is $m$-maximal, so Lemma 3 implies that $P \subseteq F(G)$. This being true for all Sylow subgroups of $G$, we obtain $G=F(G)$, which means that $G$ is nilpotent. 
THEOREM 7. Let each n-maximal subgroup of $G$ be subnormal. Suppose that $w(|G|) \geqq n-k$ and let $P$ be any Sylow subgroup of $G$. If $P$ is not normal in $G$, then $P$ has a cyclic l-maximal subgroup, for some $l \leqq k$.

Proof. If $P$ is subnormal, then, being a Sylow subgroup, it is normal in $G$. Suppose $P$ is not subnormal, and construct a chain $P=H_{0} \subset H_{1} \subset \ldots \subset H_{m}=G$ as in the preceding proof. Then, as above, we find that $m \geqq n-k-1$.

Let $H$ be a subgroup of $P$, minimal with respect to not being subnormal in $G$. $H$ is $l$-maximal in $P$, for some $l$, and hence $(l+m)$-maximal in $G$. Lemma 2 implies $l+m<n$, which, together with $m \geqq n-k-1$, implies $l \leqq k$.

Now each maximal subgroup of $H$ is subnormal in $G$, but $H$ is not, and so $H$ cannot be generated by its maximal subgroups. This is possible only if $H$ has only one maximal subgroup, and this implies that $H$ is a cyclic $p$-group, which concludes our proof.

REMARK. In exactly the same way we can prove: if $K$ is a subgroup of $G$, such that $|G: K|$ is divisible by $n-k-1$ distinct primes at least, then $K$ is subnormal, or has a cyclic $l$-maximal $p$-subgroup, for some $l \leqq k$.

Taking $k=0$ in the last theorem, we obtain

COROLlaRY 2. If each n-maximal subgroup of $G$ is subnormal, and $w(|G|) \geqq n$, then each Sylow subgroup of $G$ is either cyclic or normal.

THEOREM 8. If each n-maximal subgroup of $G$ is subnormal and $w(|G|) \geqq n$, then $G$ is either of the following two types:

(a) $G$ is nilpotent.

(b) $G=H N$, where,

(i) $N$ is a normal abelian Hall subgroup, and all Sylow subgroups of $N$ are elementary abelian.

(ii) $H$ is a cyclic Hall subgroup, and $|H|$ is either a prime-power or a square-free number.

(iii) $(|N|,|H|)=1$.

(iv) If $H_{p}$ is a Sylow subgroup of $H$, and $N_{q}$ is a Sylow subgroup of $N$, then $H_{p}$ induces in $N_{q}$ an irreducible automorphism group of order $p$ or 1 . In the latter case $\left|N_{q}\right|=q$.

Conversely, a group of type (a) or (b) has each n-maximal subgroup subnormal.

Proof. By Corollary 2, each Sylow subgroup of $G$ is either normal or cyclic. Assume $G$ is not nilpotent. Then at least one Sylow subgroup is not normal. If $|G|$ is square-free, our result follows from the structure theorem for such groups [5, Theorem 9.4.3]. Hence, we shall suppose $|G|$ is not square-free.

Let $G_{q_{1}}, \ldots, G_{q_{r}}$ be the normal Sylow subgroups of $G$, and let $N=G_{q_{1}} \cdots G_{q_{r}}$. Then $N$ is nilpotent, and $N \neq G$. If $N=1$, all Sylow subgroups of $G$ are cyclic, but then $G$ does have a normal Sylow subgroup [5, Theorem 9.4.3]. So $N \neq 1$ always. Let $H$ be a Hall subgroup of $G$ of order $|G: N|$; then $G=H N$ and $(|H|,|N|)=1$. Also 
each Sylow subgroup of $H$ is cyclic. Using again [5, Theorem 9.4.3] we find that $H$ has a Sylow tower,

$$
1 \triangleleft H_{p_{1}} \triangleleft H_{p_{1}} H_{p_{2}} \triangleleft \cdots \triangleleft H_{p_{1}} H_{p_{2}} \cdots H_{p_{\mathrm{s}}}=H,
$$

where $H_{p_{1}}, \ldots, H_{p_{s}}$ are the Sylow subgroups of $H$ in some order. By definition of $N$ and $H$, none of the $H_{p_{i}}$ 's is normal in $G$.

Let $H_{p}$ be any Sylow subgroup of $H$, and consider the chain

$$
H_{p} \subseteq H_{p} G_{q_{1}} \subseteq H_{p} G_{q_{1}} G_{q_{2}} \subseteq \cdots \subseteq H_{p} N \subseteq H_{p} N H_{p_{1}} \subseteq H_{p} N H_{p_{1}} H_{p_{2}} \subseteq \cdots \subseteq G .
$$

If $p=p_{i}$, then $H_{p} N H_{p_{1}} \cdots H_{p_{i-1}}=H_{p} N H_{p_{1}} \cdots H_{p_{i}}$. All the other containments in (S2) are proper. Since $w(|G|)=n$ (otherwise $G$ is nilpotent) there are $n$ distinct terms in (S2). $H_{p}$ is not normal, therefore not subnormal; therefore, by Lemma 2 , $H_{p}$ is not $k$-maximal for any $k \geqq n$. This implies, in particular, that the chain (S2) cannot be further refined. This implies, in the first place, that $H_{p}$ acts irreducibly on every $G_{q_{i}}$, which gives (i) and part of (iv). If $M$ is a maximal subgroup of $H_{p}$, then $M$ is $n$-maximal, as is shown by (S2), so $M$ is subnormal in $G$, and also in $M N$. However, $M$ is a Sylow subgroup of $M N$, so $M \triangleleft M N, M N=M \times N$, and $M$ centralizes $N$. This gives the rest of (iv) (the last statement of (iv) is an immediate consequence of the first).

The fact that (S2) cannot be further refined implies also that (S1) cannot be further refined, except perhaps in the link $H_{p_{1}} \cdots H_{p_{i-1}} \subseteq H_{p_{1}} \cdots H_{p_{i}}$, where $p=p_{i}$. This is possible only if $\left|H_{p_{j}}\right| \leqq p_{j}$, for $p_{j} \neq p$. If $H \neq H_{p}$, then starting from some other Sylow subgroup of $H$, we find that also $\left|H_{p}\right|=p$ and $|H|$ is square-free.

We have proved by now everything but the fact that $H$ is cyclic. This is obvious if $|H|$ is a prime-power, since each Sylow subgroup of $H$ is cyclic. Suppose $H$ has a square free order, and is not cyclic. Then there must be two Sylow subgroups of $H$ which do not commute elementwise, otherwise $H$ is the direct product of its cyclic Sylow subgroups, and is cyclic. There exists, therefore, a nonabelian Hall subgroup $K$ of $H$ of order $p_{1} p_{2}$, say. $|G|$ was supposed not to be square free, while $|H|$ is square-free, so $\left|G_{q}\right| \neq q$, for some $q|| N \mid$. By (iv), each of $H_{p_{1}}, H_{p_{2}}$ acts irreducibly on $N_{q}$, and does not centralize it. Hence, $K$ is a group of fixed-point-free automorphisms of $N_{q}$, having order $p_{1} p_{2}$. According to Burnside [2, p. 335] $K$ is cyclic, a contradiction.

To prove the converse, let $G$ be any group of type (a) or (b). In case (a) each subgroup of $G$ is subnormal. Let $G$ be of type (b). We may assume that $N$ contains every normal Sylow subgroup of $G$. $N$ being abelian, any subgroup of $N$ is normal in $N$ and therefore subnormal in $G$.

Let $K$ be any subgroup of $G$ which is not subnormal. Then $K \notin N$; therefore $|K|$ and $|H|$ have a common prime divisor, $p$ say. If $|H|$ is square-free, any Sylow $p$-subgroup of $K$ is also a Sylow $p$-subgroup of $G$. If $|H|$ is not square-free, $H$ is a $p$-group and $H^{p}$ centralizes $N$, by (iv). Since $H^{p} \triangleleft H$ and $G=N H$, this implies $H^{p} \triangleleft G$. Therefore also $N \times H^{p} \triangleleft G$, and $N \times H^{p}$ is a normal abelian subgroup of $G$. Hence, 
$K \nsubseteq N \times H^{p}$. Since $G=N H$ and $H$ is cyclic, this implies that a Sylow $p$-subgroup of $K$ is again a Sylow $p$-subgroup of $G$.

Now consider any maximal chain

$$
K=K_{0} \subset K_{1} \subset \ldots \subset K_{m}=G .
$$

Let $q$ be prime divisor of $|G: K|$, and let $i$ be the first index such that $q|| K_{i+1}: K_{i} \mid$. If $q|| H \mid$, then, since $q \neq p,|H|$ must be square-free, and $q$ is the maximal power of $q$ dividing $|G|$. If $q|| N \mid$, then, since $G$ is solvable and $K_{i}$ is maximal in $K_{i+1}$, $\left|K_{i+1}: K_{i}\right|=q^{j}$. Let $Q$ be a Sylow $q$-subgroup of $K_{i+1}$, then $K_{i+1}=K_{i} Q$ and $Q \subseteq N_{q}$ ( $N_{q}$ being the unique Sylow $q$-subgroup of $G$ ). Also $Q \triangleleft K_{i+1}$ and $K \subseteq K_{i+1}$. Since $K$ contains a Sylow $p$-subgroup of $G$, and this last acts irreducibly on $G_{q}$, we must have $Q=G_{q}$. This implies that $q^{j}$ is the maximal power of $q$ dividing $|G: K|$.

It is obvious from the former considerations, that any prime divisor of $|G: K|$ divides exactly one of the numbers $\left|K_{i+1}: K_{i}\right|$. Remembering that $K$ contains a Sylow subgroup of $G$, it follows that (S3) has at most $n$ terms, where $n$ is the number of distinct prime divisors of $|G|$. Hence $K$ is $(n-1)$-maximal at most, and any $n$ maximal subgroup of $G$ is subnormal. This ends the proof of Theorem 8 .

The existence problem for nonnilpotent groups satisfying the condition of Theorem 8 is solved by means of the following result of Miller and Moreno [12, pp. 400-402].

Let $p$ and $q$ be given prime numbers $(p \neq q)$; then there exists, up to isomorphism, exactly one group $S$ of order $p q^{m}$, in which the Sylow q-subgroup is a minimal normal subgroup, and with trivial center. For this group, $m$ is the order of $q(\bmod p)$.

Now suppose two sets of primes, $\left\{q_{1}, \ldots, q_{r}\right\}$ and $\left\{p_{1}, \ldots, p_{s}\right\}$ are given. We want to construct a nonnilpotent group $G$, of type (b) in Theorem 8 , such that $\left\{q_{1}, \ldots, q_{r}\right\}$ is the set of prime divisors of $|N|$, while $\left\{p_{1}, \ldots, p_{s}\right\}$ is the corresponding set for $|H|$. We always take $N$ to contain all the normal Sylow subgroups of $G$.

First suppose $|H|=p_{1}$. Either $H$ centralizes $N_{q}$, where $q \in\left\{q_{1}, \ldots, q_{r}\right\}$, or $H N_{q}$ is the group described in the Miller-Moreno theorem (with $p=p_{1}$ ). $H$ cannot centralize all the $N_{q}$ 's, for if it did $H$ would be normal. Hence, there are exactly $2^{r}-1$ possibilities for the action of $H$ on $N$, and the same number of groups $G$ with $|H|=p_{1}$.

For any natural number $m$, we also have $2^{r}-1$ possibilities for groups $G$ with $|H|=p_{1}^{m}$. Here $H$ is cyclic, $H^{p_{1}} \subseteq Z(G)$, and $G / H^{p_{1}}$ has the structure described in the former paragraph.

Now let $s>1$. Then we must have $|H|=p_{1} \cdots p_{s}$. For any $p_{i}$ and $q_{j}, H_{p_{i}} N_{q_{j}}$ is either cyclic of order $p_{i} q_{j}$ or is again the group described by Miller and Moreno. $H_{p_{i}}$ cannot centralize all the $N_{q_{j}}$ 's, or it would be normal. Hence, denoting by $\tau_{i}$ the subset of $\left\{q_{1}, \ldots, q_{r}\right\}$ consisting of primes $q_{j}$ such that $H_{p_{i}} N_{q j}$ is noncyclic, the sets $\tau_{i}$ must satisfy

(i) $\tau_{i}$ is nonempty.

(ii) If $q_{j} \in \tau_{i} \cap \tau_{k}, q_{j}$ has the same order $\left(\bmod p_{i}\right)$ and $\left(\bmod p_{k}\right)$.

((ii) follows from the last statement of the Miller-Moreno result.) 
For any system of subsets $\tau_{1}, \ldots, \tau_{s}$ of $\left\{q_{1}, \ldots, q_{r}\right\}$, there corresponds exactly one group $G$, and the number of such groups is equal to the number of such systems. In particular, if $s \leqq r$, we can always choose disjoint $\tau_{i}$ 's, so (ii) is automatically fulfilled. For $s>r$, however, it is possible that no group $G$ exists.

Next, we consider the case in which $|G|$ is divisible by $n-1$ distinct primes.

THEOREM 9. Let each n-maximal subgroup of $G$ be subnormal. If $w(|G|) \geqq n-1$, then each Sylow subgroup of $G$ is either normal or of one of the following types:

(i) Cyclic.

(ii) A direct product of a cyclic group and a group of prime order.

(iii) The group $\left\langle a, b \mid a^{p^{m-1}}=b^{p}=1, b^{-1} a b=a^{1+p^{m-2}}\right\rangle$, $p$ a prime.

(iv) The quaternion group.

Proof. Suppose $P$ is a nonnormal Sylow subgroup of $G$. By Theorem 7, $P$ has a maximal cyclic subgroup. We have seen in the proof of Theorem 7 , that $P$ is $n$-2-maximal at least, so each 2nd-maximal subgroup of $P$ is $n$-maximal at least, and is subnormal, by Lemma 2 . But $P$ is not subnormal, so $P$ is not generated by its 2nd-maximal subgroups. Working through the list of $p$-groups with maximal cyclic subgroups [e.g. 5, Theorem 12.5.1], we find that the only ones not generated by their 2nd-maximal subgroups are those mentioned in Theorem 9.

THEOREM 10. Let $G$ be as in Theorem 9. Then $G$ has a Sylow tower.

Proof. The conditions imposed on $G$ are preserved in homomorphisms (possibly with a smaller $n$ ). Therefore, it suffices to show that $G$ has a normal Sylow subgroup. Suppose this is not the case. Then, by Theorem 9, all Sylow subgroups of $G$ have cyclic maximal subgroups. Let $M / N$ be any chief factor of $G$. Then $M / N$ is a $p$-group, for some prime $p$. Then $M / N$ is elementary abelian, on one hand, and has a cyclic maximal subgroup, on the other hand, so $|M / N| \leqq p^{2}$. This means that the rank of $G$ is at most 2. Since $G$ does not have a normal Sylow subgroup, a theorem of Huppert [7, Satz 14] forces $|G|=2^{\alpha} 3^{\beta}$. The assumption on $|G|$ now implies $n \leqq 3$, and we must have $n=3$, or Theorem 8 applies.

Let $F=F(G)$. By Lemma 3, each 3-maximal subgroup of $G / F$ is trivial. However, $G / F$, being solvable, has a chain of subgroups such that each one has a prime index in its successor (e.g. a composition series). This shows that $|G / F|$ is a product of three primes at most. If $|G / F|$ is a prime-power, then the Sylow subgroup belonging to the other prime dividing $|G|$ is contained in $F$, and hence is normal in $G$. That leaves the possibilities: $|G / F|=6,12$ or 18 .

If $|G / F|=12$ or $18, G / F$ has a subgroup $K / F$, which is subnormal, of prime order, and 2nd-maximal. $K$ is then a subnormal 2nd-maximal subgroup of $G$, which is nilpotent by Lemma 1 . Hence, $K \subseteq F$, a contradiction.

So $|G / F|=6$. Suppose $F_{3} \neq 1$, and let $a$ be an element of $G_{2}$ outside $F$. Consider the chain $\langle a\rangle \subset\langle a\rangle F_{3} \subseteq G_{2} F_{3} \subset G$. $\langle a\rangle \nsubseteq F$ implies $\langle a\rangle \triangleleft \nsucc G$, hence $\langle a\rangle$ is not 3-maximal which forces $\langle a\rangle=G_{2}$. Now $G_{2}$ is cyclic, and $G$ has a normal Sylow 3-subgroup, by Burnside's Theorem [5, Theorem 14.3.1]. 
Therefore, $F_{3}=1$. Now consider the chain $G_{3} \subset G_{3} F_{2} \subset G$. As above, $G_{3}$ is not 3-maximal, so this chain cannot be refined. This says that $G_{3}$, of order 3 , is irreducible on $F_{2}$, so $\left|F_{2}\right|=2$ or 4 . If $\left|F_{2}\right|=2$, then $|G|=12$, and $G$ has a normal Sylow subgroup. If $\left|F_{2}\right|=4$, then $G$ is a group of order 24 possessing a self-centralizing normal subgroup of order 4 , which implies $G \cong S_{4}$ [17, p. 148]. But $S_{4}$ has 3-maximal subgroups (of order 2) which are not subnormal, a contradiction.

Let now $G$ be any group satisfying the conditions of Theorem 9. Since $G$ has normal Sylow subgroups, by Theorem 10, we can form the subgroups $N$ and $H$ as in the proof of Theorem 8 (we may assume that $G$ is not nilpotent), and consider the chain corresponding to (S2). (Notice that the chain corresponding to (S1) exists, by Theorem 10.) This can now be refined at one place at most. As in the proof of Theorem 8, we can conclude from this fact the following:

$N$ is nilpotent, and its Sylow subgroups are elementary abelian, with at most one exception. $|H|$ has the form $p_{1}^{\alpha} p_{2}^{\alpha_{2}} \cdots p_{k}^{\alpha}$, where the $p_{i}$ 's are primes, $\alpha_{1} \leqq 4, \alpha_{2} \leqq 2$, and the rest of the $\alpha_{i}$ 's are either zero or one. Any $H_{p}$ induces on any $N_{q}$ a group of order $p^{2}$ at most, and this is irreducible for all but at most one $q$.

Theorem 10 is no longer true for $n-2$ replacing $n-1$, as the symmetric group $S_{4}$ shows. However, since all $p$-groups with a cyclic 2 nd-maximal subgroup were determined [2, pp. 136-139], [10], [11], one can determine the structure of nonnormal Sylow subgroups of a group satisfying the conditions of Theorem 9, with $n-2$ replacing $n-1$, as in Theorem 9. This can also be done for $n-3$, and odd $p$, using the results of [13].

Now we impose on $G$ the stronger condition, that each $n$-maximal subgroup is quasi-normal. The main result here is

THEOREM 11. Let each n-maximal subgroup of $G$ be quasi-normal. If $w(|G|)$ $\geqq n-k+1(k \geqq 1)$, then $r(G) \leqq k$.

Proof (by induction on $|G|$ ). Let $M$ be a maximal subgroup of $G$. Then $|G: M|$ is a prime-power, so $|M|$ is divisible by $n-k=n-1+k+1$ distinct primes at least. Hence, $M$ satisfies the same assumptions as $G$, with $n-1$ replacing $n$. By induction, $r(M) \leqq k$.

Let $H$ be any proper subgroup of $G$. Then $H$ can be embedded in a maximal subgroup, so the preceding paragraph implies $r(H) \leqq k$.

Let $N$ be any minimal normal subgroup of $G$, and let $|N|=p^{m}$. If $p|| G: N \mid$, then $G / N$ satisfies the same assumptions as $G$, so $r(G / N) \leqq k$ by induction. If $p \nmid|G: N|$, then $G / N$ is isomorphic to a $p$-complement of $G$; hence $r(G / n) \leqq k$ by the preceding paragraphs.

Lastly, let $G / K$ be any proper factor-group of $G$. Then $G / K$ is a homomorphic image of $G / N$, for some minimal normal subgroup $N$, hence $r(G / K) \leqq k$.

$r(G) \leqq k$ is equivalent, by a result of Huppert [7, Satz 1], to the following: If $H$ is a subgroup of $G, K$ is a maximal subgroup of $H$, and $|H: K|=p^{m}$, with $p$ a prime, then $m \leqq k$. If $H$ is a proper subgroup, this inequality follows from the same result 
and the already proven fact $r(H) \leqq k$. So it suffices to show: if $M$ is a maximal subgroup of $G$, and $|G: M|=p^{m}, p$ a prime, then $m \leqq k$. If $M$ contains a proper normal subgroup of $G, N$, then $M / N$ is maximal in $G / N$, and $|G / N: M / N|=|G: M|$, so $m \leqq k$ follows from $r(G / N) \leqq k$. This proves our result for the case that each maximal subgroup of $G$ contains a nonidentity normal subgroup of $G$.

We may assume, then, that $G$ has a maximal subgroup $M$ with $M_{G}=1$. By Lemma 4, $M$ contains no nonidentity subnormal subgroup, and in particular, no nonidentity quasi-normal subgroup. That means that all $(n-1)$-maximal subgroups of $M$ are trivial. $M$ being solvable, this is possible only if $|M|$ is a product of $n-1$ primes at most. On the other hand, since $|G: M|$ is a prime-power, the condition on $|G|$ implies $w(|M|) \geqq n-k$.

Let $N$ be any minimal normal subgroup of $G$, and let $|N|=p^{m}, p$ a prime. Then $N \cap M=1$ and $G=N M$, so $G / N \cong M$. Form a composition series of $G$ passing through $N$, and let $G_{n}$ be the $n$th term of this series. Since $|G: N|$ has less than $n$ prime factors, $G_{n} \subset N$. Also, $G_{n}$ is quasi-normal; hence $M G_{n}$ is a subgroup, and $\left|M G_{n}\right|=|M|\left|G_{n}\right|<|M||N|=G$. Maximality of $M$ forces $M G_{n}=M$ and $G_{n}=1$. On the other hand, $|G: N|$ having at least $n-k$ prime factors, there are at most $k$ terms of our composition series between $N$ and $G_{n}$. Hence $|N|=\left|N: G_{n}\right| \leqq p^{k}$.

Now form any chief series through $N$. We have seen that $|N|=p^{m}$, with $p$ a prime and $m \leqq k$. The corresponding fact for the other factors in the series follows from $r(G / N) \leqq k$. Hence $r(G) \leqq k$.

COROllary 3. If each n-maximal subgroup of $G$ is quasi-normal ( $n \geqq 1)$, then $r(G) \leqq n-1$.

For $n \leqq 4$ (and with normality rather than quasi-normality) this is due to Huppert [7, Sätze 23, 24] and Janko [8, Theorem 4].

Proof. If $|G|$ has at least two distinct prime factors, this is obtained by substituting $k=n-1$ in the preceding theorem. If $|G|$ is a prime-power, $r(G)=1$.

Suppose that each $n$-maximal subgroup of $G$ is quasi-normal, and that $w(|G|)=n$. If $G$ is nilpotent, it is of type (b) in Theorem 8. Moreover, $r(G)=1$ by Theorem 11 . Since each Sylow subgroup of $N$ (in the notation of Theorem 8 ) is a minimal normal in $G,|N|$ is square-free.

Conversely, assume $G$ is a group of type (b) in Theorem 8, with $|N|$ square-free. The proof of the inverse part of Theorem 8 actually shows that each $n$-maximal subgroup, $K$, of $G$ is contained in $N$, if $|H|$ is square-free, and in $N \times H^{p}$, if $|H|=p^{m}$. Now $K$ is nilpotent, so a direct product of its Sylow subgroups. These Sylow subgroups are either Sylow subgroups of $N$, which are normal in $G$, or a subgroup of $H^{p}$. However, $H^{p} \triangleleft H$ (see proof of Theorem 8), and each subgroup of the cyclic group $H^{p}$ is characteristic, hence again normal in $G$. So, for such a group $G$, each $n$-maximal subgroup is even normal (if $|H|$ is square-free, each $n$-maximal subgroup is the identity).

We can also improve Theorem 10. Thus, if each $n$-maximal subgroup of $G$ is 
quasi-normal, and $|G|$ is divisible by $n-1$ distinct primes, Theorem 11 implies $r(G) \leqq 2$. According to Huppert [7, Satz 14], if $p$ is the largest prime dividing $|G|$, $G_{p} \triangleleft G$, unless perhaps $p=3$. Combined with Theorem 10 , this shows that $G$ has a Sylow tower, which is ordered, except that the Sylow 2-subgroup may precede the Sylow 3-subgroup (this actually occurs; consider $A_{4}$ ).

Lastly, we remark that the Miller-Moreno groups, mentioned on page 402, show that the analogue of Theorem 11 for subnormal subgroups is false. Here $|G|=p q^{m}$, each 2nd-maximal subgroup of $G$ is subnormal, and $r(G)=m$. However, for appropriate $p$ and $q, m$ may be arbitrarily large.

\section{Not necessarily solvable groups with subnormal $n$-maximal subgroups.}

THEOREM 12. Let $G$ be a finite group, and let $n$ and $r$ be natural numbers. Suppose

(a) Each n-maximal subgroup of $G$ is subnormal.

(b) If $H$ is a subgroup of $G$, and $K$ is maximal in $H$, then $w(|H: K|) \leqq r$.

(c) $w(|G|) \geqq(n-3) r+2$.

Then $G$ is solvable, or else $n=4, r=3$ and $G \cong \operatorname{PSL}(2, p)$, for some prime $p$.

Proof. If $n \leqq 3$, Corollary 1 shows that $G$ is solvable. Hence, we shall assume $n \geqq 4$. We have to distinguish certain possibilities for $n$ and $r$.

I. $r=1$. In that case our assertion is that $G$ is solvable, and it will be proved by induction on $|G|$.

Let $H$ be any maximal subgroup of $G$. Then $H$ satisfies the same conditions, with $n-1$ replacing $n$. $H$ is solvable, by induction, so each proper subgroup of $G$ is solvable.

Let $N$ be a minimal normal subgroup of $G$. Being solvable, $N$ is a $p$-group, $|N|=p^{m}$, say. If $p|| G / N \mid, G / N$ is solvable by induction. If $p \nmid|G / N|, G / N$ is isomorphic to a subgroup of $G$, by Schur's splitting theorem [5, Theorem 15.2.2], so is solvable by the previous paragraph. In any case $G / N$ is solvable; so, if $N$ exists, $G$ is solvable. Therefore we may assume that $G$ is simple.

Let $H$ be a maximal subgroup of $G$. Then each (n-1)-maximal subgroup of $H$ is subnormal, and therefore is the identity, as $G$ is simple. Since $H$ is solvable, this means that $\Omega(|H|) \leqq n-1$. On the other hand, (b) and (c) imply $w(|H|) \geqq n-2$. Hence $|H|$ is either square-free, or $|H|=p_{1}^{2} p_{2} \cdots p_{n-2}$, where the $p_{i}$ 's are primes. Let $P$ be any Sylow $p$-subgroup of $G$. Then $P$ can be embedded in a maximal subgroup of $G, H$, so $|P||| H \mid$, implying $|P|=p$ or $|P|=p^{2}$. Hence $|G|$ is cubefree.

Let again $H$ be any maximal subgroup. By assumption, $|G: H|$ is prime-power. By the previous arguments, $|G: H|$ is a prime or a square of a prime. Now [5, Theorem 10.5.7] implies that $G$ is solvable.

From now on we assume $r \geqq 2$.

II. $n=4$. If $G$ is nonsolvable, an already mentioned result of Janko [8, Theorems $1,2]$ implies that $G$ is isomorphic to $\operatorname{PSL}(2, p)$, for some prime $p>3$, or to $\operatorname{SL}(2,5)$. 
$|G|$ is divisible by at least $r+2 \geqq 4$ distinct primes, and this rules out $\operatorname{SL}(2,5)$, of order 120.

In order to determine the indices of maximal subgroups we need the following facts.

The group PSL $(2, q)$ always has dihedral subgroups of order $q+1, q-1$ ( for $q$ odd), or $2(q+1), 2(q-1)$ (for $q$ even). These subgroups are maximal, with the following exceptions: $q=7,9$ for the order $q+1 ; q=2$ for $2(q-1) ; q=3,5,7,9,11$ for $q-1$.

This is proved by inspecting Dickson's list of subgroups of $\operatorname{PSL}(2, q)[3, \mathrm{pp}$. 285-286].

In our case, the values $q=2,3$ are ruled out since the corresponding groups are solvable. For $q=5,7,9$, the order of $\operatorname{PSL}(2, q)$ has only three different prime divisors, and PSL $(2,11)$ contains properly the group $A_{5}$, hence it has nontrivial 4-maximal subgroups. We conclude that $G \cong \operatorname{PSL}(2, p)$, and $G$ has maximal subgroups of orders $p+1, p-1$. The indices of these maximal subgroups are $\frac{1}{2} p(p-1)$ and $\frac{1}{2} p(p+1)$. One of the numbers $p-1, p+1$, say $p+\varepsilon$, has the form $4 k$. Here $k \neq 1,2$, because the possibilities $p=3,5,7,9$ have been ruled out already. However, $k$ must be a prime, since each 3-maximal subgroup of the group of order $4 k$ is trivial. Therefore, $G$ has a maximal subgroup with index $2 p k$, so $r \geqq 3$.

$p-\varepsilon=2 l, l$ odd. Here $l$ is a product of two primes at most. The order of $G$ is $\frac{1}{2} p(p-1)(p+1)=4 p k l$. Since $r \geqq 3$, this number must have at least five distinct prime divisors. $k$ being prime, this forces $l$ to be a product of two (distinct) odd primes. We now find (using Dickson's list and the fact that all proper subgroups of $G$ are solvable), that $r=3$ and $|G|$ has exactly $(n-3) r+2=5$ distinct prime divisors.

III. $n=5$. Here we prove that $G$ is solvable, by induction on $|G|$.

Suppose some proper subgroup $H$ of $G$ is nonsolvable. Then the previous case shows that $H \cong \operatorname{PSL}(2, p), w(|H|)=5$, and $r \geqq 3$, which implies $w(|G|) \geqq 8$. Hence $w(|G: H|) \geqq 3$.

Let $N$ be a proper normal subgroup. If $N$ is nonsolvable, it is necessarily maximal, so $|G: N|$ is a prime, contradicting the previous paragraph. Hence $N$ is solvable. In particular, if $N$ is a minimal normal subgroup, $|N|=p^{m}, p$ a prime. If $p|| G: N \mid, G / N$ satisfies all our requirements, is solvable by induction, and so $G$ is solvable. If $p \nmid|G: N|$, Schur's splitting theorem yields $G=H N, H \cap N=1$. $H$ cannot be nonsolvable since $|G: H|=p^{m}$. Since $G / N \cong H$, we again find that $G$ is solvable.

So we may assume that $G$ is simple. We could now use Janko's theorem (see Theorem 5) to show that $G \cong \operatorname{PSL}(2, q)$. However, in order to make the proof more elementary (in particular, to avoid the Feit-Thompson theorem, which enters into Janko's proof), we prefer to argue as follows. First, if $H$ is a 2-maximal subgroup of $G, w(|H|) \geqq 2$. Hence, each Sylow $p$-subgroup of $G$ is at least 3-maximal. Since each 5-maximal subgroup is the identity, the order of such a Sylow subgroup is $p^{2}$ at most. A result of Burnside [5, Corollary 14.3.1] shows that 12||$G \mid$. 
Let $G_{2}$ be a Sylow 2-subgroup of $G$. Since the Sylow 2-subgroup of a simple group is not cyclic, $G_{2}$ is the four-group. $N\left(G_{2}\right) / G_{2}$ has a cube-free odd order, so the same theorem of Burnside implies that it is a solvable group, and so $N\left(G_{2}\right)$ is solvable. This implies $\Omega\left(\left|N\left(G_{2}\right)\right|\right) \leqq 4$, and since 4||$N\left(G_{2}\right) \mid, w\left(\left|N\left(G_{2}\right)\right|\right) \leqq 3$. If $N\left(G_{2}\right)$ is maximal in $G, w\left(\left|N\left(G_{2}\right)\right|\right) \geqq r+2 \geqq 4$, an impossibility. Therefore $N\left(G_{2}\right)$ is at least 2-maximal, and $\Omega(|N(G)|) \leqq 3$. $G_{2}$ is not central in $N\left(G_{2}\right)$, because $G$ is simple [5, Theorem 14.3.1]. That leaves us the only possibility $\left|N\left(G_{2}\right)\right|=4 p, p$ a prime, and $G_{2}=C\left(G_{2}\right)$. Now we may apply the corollary on p. 554 of [4] to conclude that $G \cong \operatorname{PSL}(2, q)$, for some odd $q$.

$G$ contains dihedral subgroups of orders $q+1, q-1$, which shows $\Omega(q-1) \leqq 4$, $\Omega(q+1) \leqq 4$. Also, $q-1$ and $q+1$ have the common prime divisor 2 , so the order of $G, \frac{1}{2}(q-1)(q+1)$, satisfies $w(|G|) \leqq 7$. The remarks at the beginning of case III show that every proper subgroup of $G$ is solvable. In particular, letting $H$ be a maximal subgroup, $\Omega(|H|) \leqq 4$, since 4-maximal subgroups in $H$ are trivial, and $w(|H|) \geqq r+2 \geqq 4$, by the assumptions of the theorem. Therefore $|H|$ is square-free. Since each Sylow subgroup of $G$ is contained in some maximal subgroup, $|G|$ is square-free, and $G$ is solvable [5, Theorem 9.4.3].

IV. $n>5$. We want to show that $G$ is solvable. If $H$ is a maximal subgroup of $G$, induction or the case $n=5$ shows that $H$ is solvable. If $G$ is nonsolvable repetition of the argument in the previous case shows that $G$ is simple. But then each $n$ maximal subgroup of $G$ is the identity. If $H$ is as above, we find $\Omega(|H|) \leqq n-1$ on the one hand, and $w(|H|) \geqq(n-4) r+2 \geqq n-4+r+2 \geqq n$ on the other hand, a contradiction.

We note, that in the exceptional case $G \cong \operatorname{PSL}(2, p)$, we have seen that $w(|G|)$ is exactly $(n-3) r+2$. This yields immediately

COROllary 4. Let $G$ satisfy assumptions (a) and (b) of Theorem 12. If $w(|G|)$ $\geqq(n-3) r+3, G$ is solvable.

We remark that Theorem 12 could probably be improved, by using deep recent results on classification of special types of simple groups.

\section{REFERENCES}

1. R. Baer, Classes of finite groups and their properties, Illinois J. Math. 1 (1957), 115-187.

2. W. Burnside, Theory of groups of finite order, 2nd ed., Dover, New York, 1955.

3. L. E. Dickson, Linear groups, Dover, New York, 1958.

4. D. G. Gorenstein and J. H. Walter, On finite groups with dihedral Sylow 2-subgroups, Illinois J. Math. 6 (1962), 553-593.

5. M. Hall, The theory of groups, Macmillan, New York, 1959.

6. I. N. Herstein, A remark on finite groups, Proc. Amer. Math. Soc. 9 (1958), 255-257.

7. B. Huppert, Normalteiler und maximale Untergruppen endlicher Gruppen, Math. Z. 60 (1954), 409-434.

8. Z. Janko, Finite groups with invariant fourth maximal subgroups, Math. Z. 82 (1963), 82-89.

9. - Finite simple groups with short chains of subgroups, Math. Z. 84 (1964), 428-437. 
10. G. A. Miller, Determination of all the groups of order $p^{m}$ which contain the abelian groups of type (m-2, 1), $p$ being any prime, Trans. Amer. Math. Soc. 2 (1901), 259-272.

11. - On the groups of order $p^{m}$ which contain operators of order $p^{m-2}$, Trans. Amer. Math. Soc. 3 (1902), 383-387.

12. G. A. Miller and H. C. Moreno, Nonabelian groups in which every subgroup is abelian, Trans. Amer. Math. Soc. 4 (1903), 398-404.

13. L. I. Neikirk, Groups of order $p^{m}$ which contain cyclic subgroups of order $p^{m-3}$, Publ. Univ. of Pennsylvania Ser. Math. No. 3, Philadelphia, Pa., 1905.

14. O. Ore, Contributions to the theory of groups of finite order, Duke Math. J. 5 (1939), 431-460.

15. I. Schur, Untersuchungen über die Darstellung der endlichen Gruppen durch gebrochene lineare Substitutionen, J. für Math. 132 (1907), 85-137.

16. M. Suzuki, The nonexistence of a certain type of simple groups of odd order, Proc. Amer. Math. Soc. 8 (1957), 686-695.

17. H. J. Zassenhaus, The theory of groups, 2nd ed., Chelsea, New York, 1958.

HeBrew UNIVERSITY,

JERUSALEM, ISRAEL

UNIVERSITY OF ILLINOIS,

URBANA, ILLINOIS 